\title{
An Integrative Literature Review of Basal-Bolus Insulin versus Sliding-Scale Insulin for Glycemic Management in the Hospitalized Non-Critically Ill Type 2 Diabetic Patient
}

\author{
Lindy HERR ${ }^{1}$ and Ladda THIAMWONG ${ }^{2, *}$ \\ ${ }^{1}$ Orlando Veterans Affairs Medical Center, Orlando, Florida, United States \\ ${ }^{2}$ College of Nursing, University of Central Florida, United States
}

('Corresponding author's e-mail: ladda.thiamwong@ucf.edu)

Received: 22 May 2017, Revised: 11 February 2018, Accepted: 26 March 2018

\begin{abstract}
Diabetes is an increasingly common chronic disease that affects the body's normal ability to control blood glucose levels due to impaired use of the hormone insulin. It is estimated that one out of every 4 adults who are hospitalized also have a diagnosis of diabetes. Diabetic inpatients face unique challenges in regards to managing their blood glucose while hospitalized due to the physiological stress of acute illness. Unfortunately, those who experience inadequate blood glucose management in the hospital are at an increased risk for poor patient outcomes, such as infection, increased length of stay, and death. There are multiple medications used to regulate blood sugar levels; however, the most commonly prescribed treatment for inpatients is the traditional sliding-scale regimen followed by the basal-bolus insulin regimen. An integrated literature review was conducted to determine if basal-bolus insulin is more effective than sliding-scale insulin in managing blood glucose levels of non-critically ill diabetic inpatients. Four well-known databases were searched and 5 relevant quantitative research articles were obtained and analyzed. The majority of the evidence supports basal-bolus insulin as the most effective treatment for managing blood glucose and preventing hyperglycemia without increasing the risk for hypoglycemia. Health care providers should order basal-bolus insulin accordingly in order to improve patient outcomes. Future research that questions why sliding-scale insulin is still widely prescribed may identify barriers related to ordering basal-bolus insulin and assist in decreasing related adverse events.
\end{abstract}

Keywords: Basal-bolus insulin, blood glucose, diabetes, glycemic control, inpatient, sliding-scale insulin

\section{Introduction}

Diabetes is a chronic condition that occurs when an individual is unable to properly produce or utilize insulin, which leads to impaired regulation of blood glucose levels [1]. In 2017, diabetes affected nearly 29.1 million people in the United States and approximately 1.4 million new cases have been recognized every year across the country [1]. The pervasiveness of diabetes in the hospitalized population is high, with an estimated 25 - $30 \%$ of inpatients considered to have a known diagnosis of the disease [2]. Diabetics who are hospitalized for any condition affecting their health also require their diabetes to be managed, which can be challenging considering the many factors that have the capability of promoting abnormal blood glucose levels, such as certain medications, acute stress, or timing of insulin administration [3]. Poor management of blood glucose levels may result in episodes of either hyperglycemia or hypoglycemia, both of which place an individual at risk for various complications [4]. Evidence supports the fact that hospitalized patients with diabetes who experience poor glycemic control are more likely to have a greater length of inpatient stay, high hospitalization costs, preventable complications, elevated incidence of infection, and even increased mortality [4]. Current guidelines 
http://wjst.wu.ac.th

suggest that the target blood glucose level associated with improved clinical outcomes for inpatients is below $180 \mathrm{mg} / \mathrm{dL}$ and below $140 \mathrm{mg} / \mathrm{dL}$ for random and fasting blood glucose levels, respectively [2].

Managing a patient blood sugar in the hospital is most often achieved by using insulin therapy [2]. The 2 main types of insulin therapy prescribed are the traditional sliding-scale regimen and the basalbolus regimen [5]. A sliding-scale insulin regimen consists of the administration of a dose of insulin based upon pre-meal blood glucose levels; research examining solely the use of sliding-scale insulin has shown that wide variations in glucose control may occur due to the nature of this type of regimen, especially because it is considered to be a non-individualized reactive approach to treating hyperglycemia [5]. Meanwhile, the basal-bolus approach provides long-acting insulin doses with bolus doses at mealtime in an attempt to mimic normal insulin levels and potentially promote tighter glycemic control [5]. Limited research has been published that compares the efficacy and safety between these 2 types of regimens [5]. The purpose of this review is to ascertain if the administration of basal-bolus insulin is more effective than the use of traditional sliding-scale insulin in managing blood glucose and preventing episodes of hyperglycemia or hypoglycemia in hospitalized adults with type 2 diabetes.

\section{Materials and methods}

In order to identify published material that examined the difference between basal-bolus insulin and sliding-scale insulin on glycemic control among non-critically ill hospitalized diabetics a thorough review of the literature was conducted. The 4 databases searched in January and February of 2017 were Cochrane Central Register of Controlled Trials, CINAHL, Health Source: Nursing/Academic Edition, and MEDLINE. The key words used in the search to retrieve relevant results were: (a) inpatient or hospitalized; (b) diabetes, diabetic, type 2; (c) basal-bolus insulin; (d) sliding-scale insulin; (e) control or management; and (f) hypoglycemia or hyperglycemia.

Results were refined to include those only written in the English language between the years 2005 and 2016. Articles that were listed multiple times in the search results were removed, and the remaining articles were scanned to ensure they satisfied the topic of interest. Literature reviews that resulted from the search were browsed to identify potential quantitative research articles that were not identified initially. The inclusion criteria were any quantitative research article that investigated the effectiveness of glycemic control as determined by a blood glucose sample among adult diabetic inpatients achieved by receiving either a sliding-scale or basal-bolus insulin regimen. Articles were excluded if they: (a) examined inpatients of long-term care facilities; (b) analyzed the use of intravenous insulin compared to subcutaneous insulin; (c) evaluated glycemic control based on hemoglobin A1c level; or (d) used computer-based programs instead of actual humans to compare insulin regimens on glycemic control. The level of evidence for the articles chosen was determined based on a rating system by Melnyk and FineoutOverholt [6], which is presented in the form of a hierarchy of evidence for intervention questions.

\section{Results and discussion}

The search conducted on the aforementioned databases resulted in a total of 31 articles that contained potentially useful information for determining whether basal-bolus insulin is superior to sliding-scale insulin in adequately managing the glucose levels of diabetic inpatients. Of the 31 articles, 10 were identified as either systematic reviews or articles that provided guidelines on glucose management, 8 articles were concerned with the use of computerized insulin clinical decision systems or insulin order sets, and 9 did not fully address the topic of interest or meet the overall inclusion criteria; as a result, 4 applicable articles were obtained. Skimming the systematic reviews for possible articles that fit the search criteria but were not found in the originally searched databases led to the discovery of one additional piece that was ultimately included in this paper. After the comprehensive literature review was completed, a total of 5 quantitative research articles were identified and analyzed to gather information on which insulin regimen allows for optimal glycemic management in the diabetic inpatient (see Table 1).

The articles included possessed quality evidence and met the criteria to be designated as level IV evidence or better based on the rating system by Melnyk and Fineout-Overholt [6]. The occurrence of 
http://wjst.wu.ac.th

hypoglycemia or hyperglycemic episodes, and the overall degree of blood glucose management were the outcomes that were evaluated when comparing the use of basal-bolus and sliding-scale insulin to determine which is more suitable for inpatient use.

Table 1 An integrative literature review of basal-bolus insulin versus sliding-scale insulin for glycemic management in the hospitalized type 2 diabetic.

\begin{tabular}{|c|c|c|c|c|c|c|c|}
\hline Citation & $\begin{array}{l}\text { Design/ } \\
\text { Methods/ } \\
\text { Level of } \\
\text { Evidence }\end{array}$ & Sample/Setting & $\begin{array}{l}\text { Major variables } \\
\text { studied and their } \\
\text { definitions }\end{array}$ & Intervention & Measurement & $\begin{array}{l}\text { Data analysis } \\
\text { and results }\end{array}$ & $\begin{array}{l}\text { Appraisal: } \\
\text { Worth to } \\
\text { practice/ } \\
\text { Limitations }\end{array}$ \\
\hline $\begin{array}{l}\text { Zaman et al., } \\
2014 \\
{[10]}\end{array}$ & $\begin{array}{l}\text { Retrospective } \\
\text { case-control } \\
\text { study. } \\
\text { Level IV }\end{array}$ & $\begin{array}{l}\text { Convenient sampling } \\
\text { method identified } 338 \\
\text { cases of patients that } \\
\text { were admitted to the } \\
\text { University of Malaya } \\
\text { Medical Centre } \\
\text { between January } 2008 \\
\text { - December } 2012 \text { with } \\
\text { diagnosis of } \\
\text { hyperglycemia. } \\
338 \text { cases divided into } \\
2 \text { groups based on } \\
\text { insulin regimen. } \\
\text { Basal-bolus insulin (n } \\
=159 \text { ) Sliding-scale } \\
\text { insulin (n = 179). } \\
\text { Patients included in } \\
\text { sample if admitted to } \\
\text { general medical unit } \\
\text { and treated with } \\
\text { insulin only during } \\
\text { admission. }\end{array}$ & $\begin{array}{l}\text { Independent variables } \\
\text { Sliding scale regimen = } \\
\text { Actrapid insulin } \\
\text { Basal-bolus regimen = } \\
\text { Actrapid and Insulatard } \\
\text { insulin } \\
\text { Dependent variables } \\
\text { Hyperglycemia = blood } \\
\text { glucose }>250 \text { mg/dL } \\
\text { Glycemic control = } \\
\text { achieved when fasting } \\
\text { plasma glucose obtained } \\
\text { in the morning was }< \\
126 \text { mg/dL or when pre- } \\
\text { meal plasma glucose } \\
\text { obtained before meals } \\
\text { was }<180 \text { mg/dL } \\
\text { Case of hypoglycemia = } \\
\text { blood glucose }<70 \\
\text { mg/dL }\end{array}$ & $\begin{array}{l}159 \text { patients } \\
\text { admitted with } \\
\text { hyperglycemia } \\
\text { were treated with } \\
\text { basal-bolus insulin } \\
\text { (mean insulin dose } \\
12.5, \mathrm{SD}=5.5 \\
\text { units) } \\
179 \text { patients were } \\
\text { treated with } \\
\text { sliding-scale } \\
\text { insulin (mean } \\
\text { insulin dose = } \\
3.14, \mathrm{SD}=0.9 \\
\text { units/hr) } \\
\text { Type of insulin } \\
\text { administered was } \\
\text { not specified. }\end{array}$ & $\begin{array}{l}\text { Glycemic control } \\
\text { was measured } \\
\text { between each of } \\
\text { the } 2 \text { insulin } \\
\text { regimen groups } \\
\text { based on their } \\
\text { fasting blood } \\
\text { glucose levels and } \\
\text { mean glucose } \\
\text { levels. } \\
\text { Measurements } \\
\text { were obtained } \\
\text { during treatment } \\
\text { according to the } \\
\text { American } \\
\text { Diabetes } \\
\text { Association } \\
\text { guidelines, no } \\
\text { specific } \\
\text { equipment listed } \\
\text { that was used to } \\
\text { obtain blood } \\
\text { glucose } \\
\text { measurement. }\end{array}$ & $\begin{array}{l}\text { T-test evaluated } \\
\text { differences in } \\
\text { means between } \\
\text { continuous data, } \\
\text { which was } \\
\text { expressed as mean } \\
\pm \text { standard } \\
\text { deviation. } \\
\text { Basal-bolus } \\
\text { insulin group had } \\
\text { significantly lower } \\
\text { fasting blood } \\
\text { glucose and mean } \\
\text { glucose levels } \\
\text { Fasting blood } \\
\text { glucose: } \\
\text { Basal-bolus }=194 \\
\pm 42 \text { mg/dL } \\
\text { Sliding-scale }= \\
210 \pm 63 \mathrm{mg} / \mathrm{dL} \\
\text { (p }=0.028 \text { ) } \\
\text { Mean glucose: } \\
\text { Basal-bolus = } 221 \\
\pm 34 \text { mg/dL } \\
\text { Sliding-scale }= \\
230 \pm 40 \mathrm{mg} / \mathrm{dL} \\
\text { (p = } 0.021 \text { ) } \\
\text { Nonsignificant } \\
\text { difference between } \\
\text { basal-bolus and } \\
\text { sliding-scale } \\
\text { groups for } \\
\text { hypoglycemic } \\
\text { cases } \\
\text { (p =0.186). }\end{array}$ & $\begin{array}{l}\text { Limitations: } \\
\text { No specific } \\
\text { device listed for } \\
\text { how data was } \\
\text { measured, } \\
\text { systematic error } \\
\text { may have resulted } \\
\text { in measurement } \\
\text { bias. } \\
\text { Cases were } \\
\text { obtained from one } \\
\text { geographical area, } \\
\text { may not provide } \\
\text { generalizable } \\
\text { results. }\end{array}$ \\
\hline $\begin{array}{l}\text { Umpierrez, } \\
\text { et al., } 2007 \\
{[8]}\end{array}$ & $\begin{array}{l}\text { Multicenter } \\
\text { randomized } \\
\text { control trial } \\
\text { Level II }\end{array}$ & $\begin{array}{l}\text { A total of } 130 \\
\text { nonsurgical inpatients } \\
\text { with an initial blood } \\
\text { glucose level of } 140 \text { - } \\
400 \mathrm{mg} / \mathrm{dL} \text { were } \\
\text { enrolled in this study. } \\
\text { Conducted at Grady } \\
\text { Memorial Hospital } \\
\text { and Jackson Memorial } \\
\text { Hospital. }\end{array}$ & $\begin{array}{l}\text { Independent variables } \\
\begin{array}{l}\text { Basal-bolus regimen }= \\
\text { glargine insulin and } \\
\text { lantus insulin }\end{array} \\
\text { Sliding scale regimen = } \\
\text { regular insulin } \\
\text { administered with meals } \\
\text { based on treatment } \\
\text { protocol }\end{array}$ & $\begin{array}{l}\text { Assigned } \\
\text { treatment protocol } \\
\text { was managed by } \\
\text { internal medicine } \\
\text { residents. Both } \\
\text { groups' oral } \\
\text { antidiabetic } \\
\text { medications were } \\
\text { stopped. } \\
\text { Basal-bolus } \\
\text { regimen patients } \\
\text { received a total }\end{array}$ & $\begin{array}{l}\text { Blood glucose } \\
\text { monitoring was } \\
\text { performed before } \\
\text { each meal and at } \\
\text { bedtime for } \\
\text { patients in both } \\
\text { insulin regimen } \\
\text { groups. If the } \\
\text { patient was } \\
\text { nothing by mouth } \\
\text { (n.p.o.) then blood } \\
\text { glucose }\end{array}$ & $\begin{array}{l}\text { Repeated- } \\
\text { measures ANOVA } \\
\text { was used to } \\
\text { analyze change in } \\
\text { blood glucose with } \\
\text { a P value }<0.05 \\
\text { considered } \\
\text { significant. Data } \\
\text { was expressed as } \\
\text { mean } \pm \text { standard } \\
\text { deviation or } \\
\text { percentage. }\end{array}$ & $\begin{array}{l}\text { No significant } \\
\text { differences in } \\
\text { mean age, race, } \\
\text { BMI, or } \\
\text { hemoglobin A1c } \\
\text { found between } \\
\text { treatment groups, } \\
\text { which suggests } \\
\text { results were not } \\
\text { influenced by } \\
\text { these confounding } \\
\text { variables. }\end{array}$ \\
\hline
\end{tabular}




\begin{tabular}{|c|c|c|c|c|c|c|c|}
\hline Citation & $\begin{array}{l}\text { Design/ } \\
\text { Methods/ } \\
\text { Level of } \\
\text { Evidence }\end{array}$ & Sample/Setting & $\begin{array}{l}\text { Major variables } \\
\text { studied and their } \\
\text { definitions }\end{array}$ & Intervention & Measurement & $\begin{array}{l}\text { Data analysis } \\
\text { and results }\end{array}$ & $\begin{array}{l}\text { Appraisal: } \\
\text { Worth to } \\
\text { practice/ } \\
\text { Limitations }\end{array}$ \\
\hline & & $\begin{array}{l}\text { Patients were } \\
\text { randomly assigned to } \\
\text { receive either sliding- } \\
\text { scale }(\mathrm{n}=65) \text { or } \\
\text { basal-bolus insulin ( } \mathrm{n} \\
=65 \text { ). } \\
\text { The mean age of both } \\
\text { groups was } 56 \text { years; } \\
\text { the mean BMI of both } \\
\text { groups was equal at } \\
32 \mathrm{~kg} / \mathrm{m}^{2} \text {. }\end{array}$ & $\begin{array}{l}\text { Dependent variables } \\
\text { Fasting glucose }=\text { pre- } \\
\text { meal blood glucose } \\
\text { measurement }(\mathrm{mg} / \mathrm{dL}) \\
\text { Random glucose }= \\
\text { measured blood glucose } \\
(\mathrm{mg} / \mathrm{dL}) \text { obtained at any } \\
\text { time during the day } \\
\text { Hypoglycemic episode } \\
=\text { blood glucose }<60 \\
\mathrm{mg} / \mathrm{dL}, \text { severe }=<40 \\
\mathrm{mg} / \mathrm{dL}\end{array}$ & $\begin{array}{l}\text { daily insulin dose } \\
\text { of glargine and } \\
\text { glulisine based on } \\
\text { admission blood } \\
\text { glucose ( } 0.4 \\
\text { units/kg for blood } \\
\text { glucose between } \\
140 \text { - } 200 \mathrm{mg} / \mathrm{dL} \\
\text { or } 0.5 \text { units } / \mathrm{kg} \text { for } \\
\text { blood glucose } \\
\text { between } 201 \text { - } 400 \\
\text { mg/dL). Half of } \\
\text { total daily dose } \\
\text { was given as } \\
\text { glargine, half was } \\
\text { divided into } 3 \\
\text { doses to be given } \\
\text { as glulisine with } \\
\text { meals. } \\
\text { Sliding scale } \\
\text { insulin group } \\
\text { received a dose of } \\
\text { regular insulin } 4 \\
\text { times a day based } \\
\text { on a protocol that } \\
\text { accounted for pre- } \\
\text { meal blood } \\
\text { glucose level. }\end{array}$ & $\begin{array}{l}\text { monitoring was } \\
\text { performed every } 6 \\
\text { h. } \\
\text { Blood glucose } \\
\text { was measured } \\
\text { using a glucose } \\
\text { meter by trained } \\
\text { staff. }\end{array}$ & 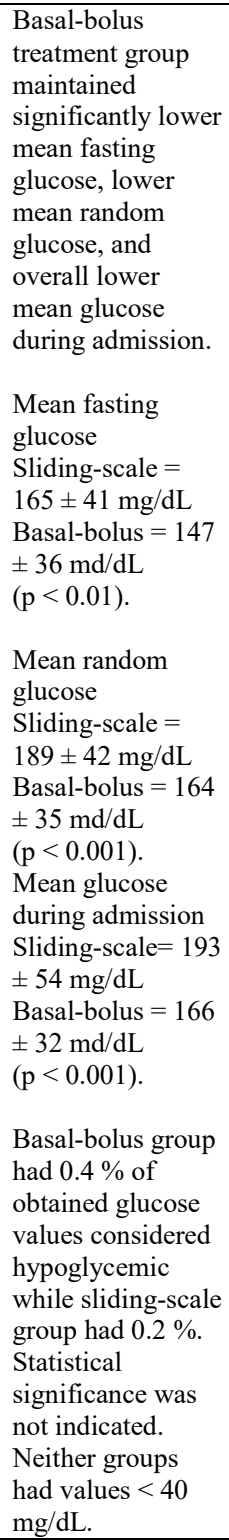 & $\begin{array}{l}\text { Basal-bolus } \\
\text { insulin may be } \\
\text { prescribed to } \\
\text { noncritically ill } \\
\text { type } 2 \text { diabetic } \\
\text { adults to manage } \\
\text { blood glucose } \\
\text { without placing } \\
\text { them at risk for } \\
\text { severe } \\
\text { hypoglycemia } \\
\text { Sliding scale } \\
\text { insulin may not } \\
\text { effectively } \\
\text { manage blood } \\
\text { glucose in } \\
\text { inpatients. } \\
\text { Limitations: } \\
\text { Patients without a } \\
\text { known history of } \\
\text { diabetes as well } \\
\text { as those that were } \\
\text { receiving } \\
\text { corticosteroids } \\
\text { were excluded, } \\
\text { which discounts } \\
\text { many hospitalized } \\
\text { diabetic patients. } \\
\text { The study did not } \\
\text { examine clinical } \\
\text { outcomes } \\
\text { between the } 2 \\
\text { treatment groups. }\end{array}$ \\
\hline $\begin{array}{l}\text { Johnston, et } \\
\text { al., } 2011 \\
{[5]}\end{array}$ & $\begin{array}{l}\text { Retrospective } \\
\text { case-control } \\
\text { study. } \\
\text { Level IV }\end{array}$ & $\begin{array}{l}45 \text { randomly selected } \\
\text { patient records from } \\
\text { the medical center at } \\
\text { University of North } \\
\text { Caroline Greensboro. } \\
\text { The patient records } \\
\text { were retrospectively } \\
\text { reviewed. } \\
\text { Sliding-scale only } \\
\text { group }(n=23)\end{array}$ & $\begin{array}{l}\text { Independent variables } \\
\text { Insulin regimen = either } \\
\text { sliding scale insulin or } \\
\text { basal-bolus insulin } \\
\text { Fasting blood glucose } \\
(\mathrm{FBS})=\text { blood glucose } \\
\text { collected between } 4-7 \\
\text { am or before breakfast }\end{array}$ & $\begin{array}{l}\text { No direct } \\
\text { intervention took } \\
\text { place, a } \\
\text { retrospective } \\
\text { review of } 23 \\
\text { patient charts from } \\
\text { the sliding-scale } \\
\text { insulin group } \\
\text { revealed they } \\
\text { received a dose of } \\
\text { regular insulin }\end{array}$ & $\begin{array}{l}\text { An electronic } \\
\text { chart } \\
\text { documentation } \\
\text { system was } \\
\text { reviewed; serial } \\
\text { blood glucose data } \\
\text { from the point-of- } \\
\text { care testing } \\
\text { database was } \\
\text { collected. }\end{array}$ & $\begin{array}{l}\text { Chi-square } \\
\text { analysis used, data } \\
\text { was presented as } \\
\text { percentages. } \\
302(56 \%) \text { out of } \\
\text { total } 540 \text { CBGs } \\
\text { collected from } \\
\text { both groups were } \\
\text { classified as } \\
\text { hyperglycemic }\end{array}$ & $\begin{array}{l}\text { Due to the fact } \\
\text { that both groups } \\
\text { experienced } \\
\text { hyperglycemic } \\
\text { events, and that } \\
\text { greater than } 50 \% \\
\text { of the data were } \\
\text { hyperglycemic } \\
\text { readings it may be } \\
\text { necessary to } \\
\text { consider dosing }\end{array}$ \\
\hline
\end{tabular}




\begin{tabular}{|c|c|c|c|c|c|c|c|}
\hline Citation & $\begin{array}{l}\text { Design/ } \\
\text { Methods/ } \\
\text { Level of } \\
\text { Evidence }\end{array}$ & Sample/Setting & $\begin{array}{l}\text { Major variables } \\
\text { studied and their } \\
\text { definitions }\end{array}$ & Intervention & Measurement & $\begin{array}{l}\text { Data analysis } \\
\text { and results }\end{array}$ & $\begin{array}{l}\text { Appraisal: } \\
\text { Worth to } \\
\text { practice/ } \\
\text { Limitations }\end{array}$ \\
\hline & & $\begin{array}{l}\text { Basal plus correction } \\
\text { group }(\mathrm{n}=22) \\
\text { Mean age } 55.6 \text { years. }\end{array}$ & $\begin{array}{l}\text { Dependent variables } \\
\text { Capillary blood glucose } \\
\text { (CBG) = sample } \\
\text { collected before meals } \\
\text { and at bedtime } \\
\text { Hypoglycemic event = } \\
\text { (CBG less than } 60 \\
\text { mg/dL } \\
\text { Hyperglycemic event }= \\
\text { CBG greater than } 180 \\
\text { mg/dL or fasting blood } \\
\text { glucose greater than } 130 \\
\text { mg/dL }\end{array}$ & $\begin{array}{l}\text { before meals } \\
\text { according to an } \\
\text { order set based on } \\
\text { their pre-meal } \\
\text { CBG. } \\
\text { Retrospective } \\
\text { review of } 22 \\
\text { patient charts from } \\
\text { the basal plus } \\
\text { correction group } \\
\text { revealed they } \\
\text { received long- } \\
\text { acting insulin in } \\
\text { the morning along } \\
\text { with correction } \\
\text { insulin with meals. } \\
\text { Specific insulin } \\
\text { dosages for each } \\
\text { group were not } \\
\text { specified in the } \\
\text { article. }\end{array}$ & $\begin{array}{l}\text { CBGs measured } \\
\text { before breakfast, } \\
\text { lunch, dinner, and } \\
\text { at bedtime were } \\
\text { examined over a } \\
\text { 3-day period. In } \\
\text { total } 540 \text { CBGs } \\
\text { recorded and } \\
\text { analyzed as data. }\end{array}$ & $\begin{array}{l}\text { events. } \\
70 \% \text { of the total } \\
\text { CBG readings } \\
\text { from the basal- } \\
\text { bolus insulin } \\
\text { group were } \\
\text { hyperglycemic } \\
\text { events, while only } \\
42 \% \text { of the total } \\
\text { CBGs from the } \\
\text { sliding scale group } \\
\text { were } \\
\text { hyperglycemic } \\
\text { events (p }<0.01) \text {. } \\
4 \text { hypoglycemic } \\
\text { events occurred in } \\
\text { sliding-scale only } \\
\text { group ( } 2 \% \text { ), none } \\
\text { occurred in the } \\
\text { basal-bolus group. } \\
\text { No significant } \\
\text { difference noted. }\end{array}$ & $\begin{array}{l}\text { insulin based on } \\
\text { carbohydrate } \\
\text { intake and } \\
\text { increasing dosing } \\
\text { regimen for } \\
\text { inpatients to } \\
\text { receive optimal } \\
\text { glycemic control. } \\
\text { Limitations: } \\
\text { Small sample } \\
\text { size, only } 45 \\
\text { charts reviewed. } \\
\text { Inpatients were } \\
\text { also prescribed } \\
\text { oral antiglycemic } \\
\text { medications, } \\
\text { which may have } \\
\text { altered their CBG } \\
\text { or FBS in ways } \\
\text { that were not } \\
\text { accounted for by } \\
\text { insulin. } \\
\text { Unmeasured } \\
\text { variables such as } \\
\text { pre-existing } \\
\text { comorbidities or } \\
\text { level of stress } \\
\text { were not } \\
\text { accounted for. }\end{array}$ \\
\hline $\begin{array}{l}\text { Rymaszew- } \\
\text { ski and } \\
\text { Breakwell, } \\
2013 \\
{[7]}\end{array}$ & $\begin{array}{l}\text { Retrospective } \\
\text { case-control } \\
\text { study } \\
\text { Level IV }\end{array}$ & $\begin{array}{l}\text { Chart review } \\
\text { conducted for patients } \\
\text { admitted to a general } \\
\text { medical floor at a } \\
\text { 140-bed teaching } \\
\text { hospital in } \\
\text { Milwaukee, WI. } \\
\text { A sample of } 128 \\
\text { charts reviewed, there } \\
\text { were } 2 \text { groups based } \\
\text { on insulin regimen. } \\
\text { Sliding-scale group (n } \\
=64 \text { ) } \\
\text { Basal-bolus group (n } \\
=64 \text { ) } \\
\text { No significant } \\
\text { differences found on } \\
\text { sex, admission blood } \\
\text { glucose, or age (SSI } \\
\text { mean age = 65.2, } \\
\text { basal-bolus mean age } \\
=63.3 \text { ). }\end{array}$ & $\begin{array}{l}\text { Independent variables } \\
\text { Sliding scale insulin = } \\
\text { pre-meal insulin dose } \\
\text { administered based on } \\
\text { pre-meal blood glucose } \\
\text { level } \\
\text { Basal-bolus insulin = } \\
\text { long acting insulin } \\
\text { administered once or } \\
\text { twice a day along with } \\
\text { nutritional dose with } \\
\text { meals } \\
\text { Dependent variables } \\
\text { Fasting blood sugar }= \\
\text { glucose level tested } \\
\text { before each meal. Goal } \\
<140 \text { mg/dL } \\
\text { Hypoglycemia } \\
\text { occurrence = blood } \\
\text { glucose level }<70 \\
\text { mg/dL }\end{array}$ & $\begin{array}{l}\text { No direct } \\
\text { intervention } \\
\text { occurred, a } \\
\text { retrospective chart } \\
\text { review was } \\
\text { conducted to study } \\
\text { the outcomes that } \\
\text { basal bolus insulin } \\
\text { compared to } \\
\text { sliding scale } \\
\text { insulin had on } \\
\text { fasting blood } \\
\text { glucose and mean } \\
\text { blood glucose. } \\
\text { The } 64 \text { patients in } \\
\text { the sliding scale } \\
\text { group had } \\
\text { received insulin } \\
\text { based on the } \\
\text { hospitals sliding } \\
\text { scale protocol (not } \\
\text { specifically } \\
\text { outlined in the } \\
\text { article). } \\
\text { The } 64 \text { patients in } \\
\text { the basal bolus } \\
\text { group received } \\
\text { insulin based on }\end{array}$ & $\begin{array}{l}\text { Electronic health } \\
\text { records were } \\
\text { reviewed to obtain } \\
\text { blood glucose } \\
\text { data. Blood } \\
\text { glucose data } \\
\text { included fasting } \\
\text { and pre-meal } \\
\text { blood glucose } \\
\text { levels that were } \\
\text { acquired before } \\
\text { meals and at } \\
\text { bedtime. The data } \\
\text { were then } \\
\text { complied to } \\
\text { determine the } \\
\text { mean. } \\
\text { The article does } \\
\text { not specify the } \\
\text { type of equipment } \\
\text { used that obtained } \\
\text { the blood glucose } \\
\text { values. }\end{array}$ & $\begin{array}{l}\text { Independent t-test } \\
\text { analyzed } \\
\text { continuous } \\
\text { variables, Mann } \\
\text { Whitney U test } \\
\text { analyzed } \\
\text { categorical data. } \\
\text { Equality of } \\
\text { variances not } \\
\text { assumed. } \\
\text { Fasting blood } \\
\text { glucose } \\
\text { significantly lower } \\
\text { with basal-bolus } \\
\text { insulin regimen. } \\
\text { Sliding-scale = } \\
201.4 \pm 33.7 \\
\text { mg/dL } \\
\text { Basal-bolus }= \\
135.5 \pm 29.9 \\
\text { mg/dL } \\
\mathrm{t}=11.71(70 \% \\
\text { CI: } 60.03,71.74) \mathrm{p} \\
<0.000 \\
\text { Mean blood } \\
\text { glucose } \\
\text { significantly lower } \\
\text { in basal-bolus }\end{array}$ & $\begin{array}{l}\text { Demographic } \\
\text { variables included } \\
\text { relevant data to } \\
\text { explain } \\
\text { occurrence of } \\
\text { hyperglycemia } \\
\text { and hypoglycemia } \\
\text { with ability of } \\
\text { being replicated. } \\
\text { Prescribing basal- } \\
\text { bolus regimen } \\
\text { may be superior } \\
\text { over sliding scale } \\
\text { insulin to } \\
\text { managing type } 2 \\
\text { inpatients } \\
\text { diabetics blood } \\
\text { glucose } \\
\text { effectively } \\
\text { without } \\
\text { increasing their } \\
\text { risk for } \\
\text { hypoglycemia. } \\
\text { Limitations: } \\
\text { Sample largely } \\
\text { African American } \\
\text { due to location, }\end{array}$ \\
\hline
\end{tabular}




\begin{tabular}{|c|c|c|c|c|c|c|c|}
\hline Citation & $\begin{array}{l}\text { Design/ } \\
\text { Methods/ } \\
\text { Level of } \\
\text { Evidence }\end{array}$ & Sample/Setting & $\begin{array}{l}\text { Major variables } \\
\text { studied and their } \\
\text { definitions }\end{array}$ & Intervention & Measurement & $\begin{array}{l}\text { Data analysis } \\
\text { and results }\end{array}$ & $\begin{array}{l}\text { Appraisal: } \\
\text { Worth to } \\
\text { practice/ } \\
\text { Limitations }\end{array}$ \\
\hline & & & & $\begin{array}{l}\text { the hospitals } \\
\text { basal-bolus insulin } \\
\text { protocol (also not } \\
\text { specifically } \\
\text { outlined). }\end{array}$ & & $\begin{array}{l}\text { group } \\
\text { Sliding-scale }= \\
225 \pm 37.5 \mathrm{mg} / \mathrm{dL} \\
\text { Basal-bolus }=149 \\
\pm 23.1 \mathrm{mg} / \mathrm{dL} \\
\mathrm{t}=13.81(70 \% \\
\mathrm{CI}: 70.25,81.71) \\
\mathrm{p}<0.000 \\
\text { No significant } \\
\text { difference between } \\
\text { the } 2 \text { groups for } \\
\text { hypoglycemia } \\
\text { occurrence. } \\
\text { Sliding-scale }= \\
0.63 \pm 0.13 \\
\text { Basal-bolus }=0.7 \\
\pm 0.13 \\
\mathrm{t}=-0.345(70 \% \\
\text { CI: }-0.31,0.16) \mathrm{p} \\
=0.73\end{array}$ & $\begin{array}{l}\text { may not be } \\
\text { applicable to } \\
\text { other racial } \\
\text { categories. } \\
\text { Noncritical units } \\
\text { not included, } \\
\text { single center } \\
\text { study, results may } \\
\text { not be } \\
\text { generalizable. } \\
\text { Possibility of } \\
\text { glucose readings } \\
\text { being obtained at } \\
\text { inappropriate } \\
\text { times due to meal } \\
\text { delivery at the } \\
\text { hospital in the } \\
\text { study; true pre- } \\
\text { meal blood sugar } \\
\text { may not have } \\
\text { always been } \\
\text { measured. }\end{array}$ \\
\hline $\begin{array}{l}\text { Umpierrez, } \\
\text { et al., } 2011 \\
\text { [9] }\end{array}$ & 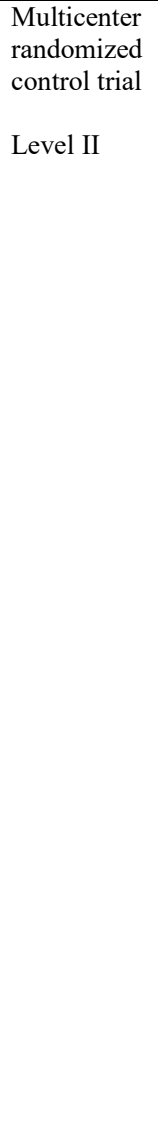 & $\begin{array}{l}\text { Patients }>18 \text { years } \\
\text { old undergoing } \\
\text { surgery and not } \\
\text { requiring ICU care } \\
\text { were eligible. } \\
\text { Conducted at Grady } \\
\text { Memorial Hospital, } \\
\text { Emory University } \\
\text { Hospital and Veterans } \\
\text { Affairs Medical } \\
\text { Center in Atlanta, } \\
\text { GA. } \\
\text { Total } 211 \text { patients } \\
\text { enrolled and randomly } \\
\text { assigned to } 2 \text { groups. } \\
\text { Sliding-scale group (n } \\
=107) \text {. } \\
\text { Basal-bolus group } \\
\text { (n=104). } \\
\text { Clinical } \\
\text { characteristics such as } \\
\text { gender, race, age, } \\
\text { BMI and body weight } \\
\text { on admission between } \\
\text { the groups were found } \\
\text { to be nonsignificant. }\end{array}$ & $\begin{array}{l}\text { Independent variables } \\
\text { Basal-bolus regimen = } \\
\text { insulins glargine and } \\
\text { glulisine } \\
\text { SSI regimen = Novolin } \\
\text { R, regular insulin } \\
\text { Dependent variables } \\
\text { Mean daily glucose } \\
\text { concentration = mean of } \\
\text { blood glucose readings } \\
\text { collected for the day, } \\
\text { goal was to maintain } \\
\text { between } 100 \text { - } 140 \\
\text { mg/dL, readings higher } \\
\text { than goal considered } \\
\text { hyperglycemic. } \\
\text { Occurrence of } \\
\text { hypoglycemia } \\
\text { (mild = blood glucose }< \\
70 \text { mg/dL or } \\
\text { severe = < } 40 \text { mg/dl). }\end{array}$ & $\begin{array}{l}\text { Patients in the } \\
\text { basal-bolus } \\
\text { regimen received a } \\
\text { total daily dose of } \\
0.5 \text { units/kg of half } \\
\text { glargine once } \\
\text { daily and half } \\
\text { glulisine (divided } \\
\text { into } 3 \text { doses to } \\
\text { receive before } \\
\text { meal times). } \\
\text { Adjustments were } \\
\text { made to the total } \\
\text { daily dose based } \\
\text { on increased age } \\
\text { or increased } \\
\text { creatinine. } \\
\text { Patients in the } \\
\text { sliding scale } \\
\text { insulin group } \\
\text { received a dose of } \\
\text { regular insulin } \\
\text { (Novolin R) } \\
\text { before meals and } \\
\text { at bedtime if their } \\
\text { pre-meal/pre- } \\
\text { bedtime blood } \\
\text { glucose was }>140 \\
\text { mg/dL. Dose of } \\
\text { insulin was } \\
\text { increased } \\
\text { according to a } \\
\text { protocol (not } \\
\text { provided in the } \\
\text { article), where the }\end{array}$ & $\begin{array}{l}\text { Mean daily blood } \\
\text { glucose } \\
\text { concentration was } \\
\text { measured for each } \\
\text { of the groups. } \\
\text { This value was } \\
\text { determined based } \\
\text { on blood glucose } \\
\text { levels that were } \\
\text { either randomly } \\
\text { obtained (at any } \\
\text { time during the } \\
\text { day) or obtained } \\
\text { before meals or } \\
\text { before bedtime. } \\
\text { Data collection } \\
\text { methods were the } \\
\text { same for both } \\
\text { groups, however } \\
\text { no specific } \\
\text { equipment stated. }\end{array}$ & $\begin{array}{l}\text { Comparison with } \\
\text { baseline and } \\
\text { outcome variables } \\
\text { completed with } \\
\text { Wilcoxon and chi- } \\
\text { square tests, data } \\
\text { presented as mean } \\
\pm \text { standard } \\
\text { deviation and } \\
\text { percentages. } \\
\text { Mean daily } \\
\text { glucose } \\
\text { concentration } \\
\text { significantly lower } \\
\text { in basal-bolus } \\
\text { group. } \\
\text { SSI = } 176 \pm 44 \\
\text { mg/dL } \\
\text { Basal-bolus }=157 \\
\pm 32 \text { md/dL (p }< \\
0.001 \text { ) } \\
\text { Fasting glucose } \\
\text { levels significantly } \\
\text { lower in basal } \\
\text { bolus group. } \\
\text { SSI = } 165 \pm 40 \\
\text { mg/dL } \\
\text { Basal-bolus }=155 \\
\pm 37 \text { mg/dL } \\
\text { (p = 0.037) } \\
\text { Mean of } 53 \% \text { of } \\
\text { patients from basal } \\
\text { bolus group met } \\
\text { goal to achieve }\end{array}$ & $\begin{array}{l}\text { Estimated } 104 \\
\text { subjects in each } \\
\text { group required to } \\
\text { achieve } 90 \% \\
\text { power, this was } \\
\text { met. } \\
\text { Inpatients } \\
\text { prescribed basal- } \\
\text { bolus achieved } \\
\text { better blood } \\
\text { glucose control } \\
\text { and lower pre- } \\
\text { meal and bedtime } \\
\text { glucose levels } \\
\text { throughout the } \\
\text { day, suggesting } \\
\text { the efficacy of } \\
\text { prescribing a } \\
\text { basal-bolus } \\
\text { insulin regimen to } \\
\text { inpatients. } \\
\text { However, patients } \\
\text { receiving basal- } \\
\text { bolus may be at } \\
\text { an increased risk } \\
\text { for hypoglycemia. } \\
\text { Limitations: } \\
\text { Factors associated } \\
\text { with } \\
\text { hypoglycemic } \\
\text { events for this } \\
\text { population may } \\
\text { have been } \\
\text { reduced intake }\end{array}$ \\
\hline
\end{tabular}


http://wjst.wu.ac.th

\begin{tabular}{|c|c|c|c|c|c|c|c|}
\hline Citation & $\begin{array}{c}\text { Design/ } \\
\text { Methods/ } \\
\text { Level of } \\
\text { Evidence }\end{array}$ & Sample/Setting & $\begin{array}{l}\text { Major variables } \\
\text { studied and their } \\
\text { definitions }\end{array}$ & Intervention & Measurement & $\begin{array}{l}\text { Data analysis } \\
\text { and results }\end{array}$ & $\begin{array}{l}\text { Appraisal: } \\
\text { Worth to } \\
\text { practice/ } \\
\text { Limitations }\end{array}$ \\
\hline & & & & $\begin{array}{l}\text { dose of insulin } \\
\text { corresponded with } \\
\text { blood glucose } \\
\text { readings. } \\
\text { Oral antidiabetic } \\
\text { medications were } \\
\text { discontinued for } \\
\text { both groups. }\end{array}$ & & $\begin{array}{l}\text { glucose reading }< \\
140 \mathrm{mg} / \mathrm{dL} \text {, while } \\
\text { only } 31 \% \text { met that } \\
\text { goal from SSI } \\
\text { group }(\mathrm{p}<0.001) \text {. } \\
\text { A significant } \\
\text { difference in the } \\
\text { occurrence of } \\
\text { hypoglycemia } \\
\text { found between the } \\
2 \text { groups. } 23.1 \% \\
\text { of patients in } \\
\text { basal-bolus group } \\
\text { experienced mild } \\
\text { hypoglycemia } \\
\text { compared to only } \\
4.7 \% \text { of patients } \\
\text { in SSI group (p }< \\
0.001) \text {. No } \\
\text { significant } \\
\text { difference found } \\
\text { for occurrence of } \\
\text { severe } \\
\text { hypoglycemia } \\
\text { between groups. }\end{array}$ & $\begin{array}{l}\text { from NPO status } \\
\text { for surgery, which } \\
\text { were not } \\
\text { accounted for. } \\
\text { The study } \\
\text { excluded patients } \\
\text { that had history of } \\
\text { hepatic disease, } \\
\text { severe renal } \\
\text { disease, or } \\
\text { hyperglycemic } \\
\text { crises, so not } \\
\text { applicable to all } \\
\text { patients. }\end{array}$ \\
\hline
\end{tabular}

\section{Hypoglycemic episodes}

All 5 articles examined the occurrence of hypoglycemia and the type of insulin regimen received by the diabetic inpatient [5,7-10]. Data presented from 4 out of 5 articles found no significant difference for the occurrence of mild to moderate hypoglycemia between sliding-scale and basal-bolus insulin groups $[5,7,8,10]$. Of note, although no significant difference in the data was found for this outcome, the research by Rymaszewski and Breakwell [7] showed the patients who received sliding-scale insulin had more hypoglycemic episodes compared to the basal-bolus group and out of the 22 patients who received basalbolus insulin in the study by Johnston and Horn [5], none of them ever experienced hypoglycemia.

The only research that presented a significant difference for mild to moderate hypoglycemia was conducted by Umpierrez et al. [9], in which 24 of the 104 patients in the basal-bolus group and only 5 of the 107 patients in the sliding-scale group experienced hypoglycemia, resulting in an absolute risk reduction of hypoglycemia to be $18 \%$ in those receiving sliding-scale insulin compared to the basal-bolus insulin group.

\section{Hyperglycemic episodes}

Two of the 5 articles reviewed specifically identified hyperglycemia as a measured outcome $[5,8]$. According to Johnston and Horn [5], hyperglycemia was defined as either a capillary blood glucose level greater than $180 \mathrm{mg} / \mathrm{dL}$ or fasting blood glucose greater than $130 \mathrm{mg} / \mathrm{dL}$ while Umpierrez et al. [8], designated hyperglycemia as any blood glucose level greater than $140 \mathrm{mg} / \mathrm{dL}$. Both articles reported statistically significant values, however the results from one study supported the use of sliding-scale insulin for prevention of hyperglycemia, while the results of Umpierrez et al. [8], found basal-bolus insulin to be more effective. Out of 276 total blood glucose samples obtained from the sliding-scale group in the study conducted by Johnston and Horn [5], 117 were hyperglycemic while 185 out of 264 from the basal-bolus group were classified as hyperglycemic. Meanwhile, $34 \%$ of patients who received basal- 
http://wjst.wu.ac.th

bolus insulin had hyperglycemia compared to $62 \%$ of those in the sliding-scale group in the research by Umpierrez et al. [8].

\section{Glycemic management}

The overall degree of blood glucose management based on the type of insulin administered was the major outcome that was examined in all 5 research articles, which all had mentioned that inadequate glycemic control is associated with poor patient outcomes [5,7-10]. Only one article found that patients receiving sliding-scale insulin achieved better glycemic control than the basal-bolus group, based on the data that depicted $41 \%$ of the subjects in the basal-bolus group had daily blood glucose levels considered inadequately controlled compared to only $19 \%$ of those in the sliding-scale group [5].

The remaining 4 articles reviewed all reported that patients who received basal-bolus insulin obtained significant improvement in glycemic control in comparison to those who were on a sliding-scale regimen [7-10]. For example, the blood glucose level on the day of discharge was significantly lower for patients receiving basal-bolus insulin in 2 studies, one of which reported a mean discharge blood glucose level of $128 \mathrm{mg} / \mathrm{dL}$ in the basal-bolus group compared to a mean discharge blood glucose of almost double or $244 \mathrm{mg} / \mathrm{dL}$ in the sliding-scale group [7,8]. Research by Umpierrez et al. [8] and Umpierrez et al. [9] investigated the mean glucose during hospital stay as a measure of glycemic control and found that the patients receiving sliding-scale insulin experienced significantly higher values than the basal-bolus group. One study had even recognized that patients who had their insulin regimen changed from slidingscale to basal-bolus demonstrated a significant improvement in glycemic control during their stay and found the mean difference in blood sugar measurements among the 2 types of therapy to be $27 \mathrm{mg} / \mathrm{dL}$ (p $<0.01)[11]$.

\section{Discussion}

Based on the evidence found in the research articles analyzed, a basal-bolus insulin regimen is more effective than the traditional sliding-scale regimen in managing blood glucose and preventing hyperglycemic or hypoglycemic episodes in the type 2 diabetic inpatient. The results suggest that target glycemic control is best achieved when patients are administered a long-acting insulin coupled with shortacting insulin, as this type of regimen closely mimics the actions of a normal pancreas [11]. The findings that sliding-scale insulin poorly controls blood glucose levels and is associated with hyperglycemia reflects the results of other published articles [12,13]. For example, Clement et al. [11] reported various concerns with the use of sliding-scale insulin due to the fact that this therapy treats high blood glucose levels once they have already occurred and fails to actually prevent the undesirable outcome. The data presented in this paper is similar to the data described by Christensen et al. [12] in a meta-analysis that also found mean daily blood glucose levels in diabetic inpatients were significantly lower among those who were receiving basal-bolus insulin compared to sliding scale insulin. In addition, Lee et al. [13] conducted a systematic review of randomized controlled trials and identified a significantly higher incidence of hyperglycemia among patients who were administered sliding-scale insulin compared to basal-bolus insulin.

Hypoglycemic episodes are considered a major safety concern for patients receiving insulin; however, the majority of the results indicate that patients receiving basal-bolus insulin are not at an increased risk for hypoglycemia. These findings are consistent with the results reported by a systematic review and meta-analysis that confirmed no significant difference for risk of severe hypoglycemia was present among patients receiving sliding-scale insulin and basal-bolus insulin [11]. Although one research article analyzed favored sliding-scale insulin for the prevention of hypoglycemia, this type of regimen has been considered to cause drastic changes in glucose levels leading to a dangerous decrease in blood glucose and a hypoglycemic event [11]. Overall, the efficacy and safety of basal-bolus insulin has been demonstrated and the collected evidence suggests that this type of insulin regimen is superior to slidingscale in managing hospitalized adults with type 2 diabetes. 


\section{Limitations of the evidence}

Each of the 5 research articles acknowledged the presence of limitations in their study. Three of the studies were retrospective in nature, which may be considered a limitation because the researchers only had access to the data that was already recorded in the patients' charts $[5,7,10]$. The applicability of the results to critical care units may not be feasible, considering both Rymaszewski and Breakwell [7] and Umpierrez et al. [9] excluded patients in their study who were cared for in the intensive care unit.

The presence of confounding variables may have led to misrepresentation of the results, as only Zaman et al. [10] took into account the use of loop diuretics and corticosteroids which are both medications commonly prescribed to inpatients that have the potential to alter blood glucose levels. Another potential confounding variable that was not considered by any of the 5 articles was the degree of physiologic stress each of the subjects was experiencing while hospitalized, which may have interfered with blood glucose levels differently for participants from each of the 2 insulin groups [5,7-9].

A specific limitation of this integrated literature review would be the quantity of articles analyzed. A total of only 5 research articles were obtained, which narrowed the amount of evidence that was examined.

\section{Conclusions and recommendations}

The majority of the evidence supports basal-bolus insulin as the most effective treatment for managing blood glucose and preventing hyperglycemia without increasing the risk for hypoglycemia. Health care providers should order basal-bolus insulin accordingly in order to improve patient outcomes.

\section{Recommendations}

\section{Practice}

Due to the fact that a larger part of the evidence reports the basal-bolus insulin regimen promotes better glycemic control without a significant concern for adverse events such as hypoglycemia, it is logical to recommend to providers to avoid ordering sliding-scale insulin over basal-bolus insulin in the diabetic inpatient. Ordering sets with a specific protocol for basal-bolus insulin may be utilized in the inpatient setting to promote the use of this type of regimen.

It is also important to appreciate the fact that nursing may play a key role in the effective management of blood glucose levels in the hospitalized type 2 diabetic patient. The nurse caring for the patient should ideally recognize instances that precipitate abnormal blood glucose levels, such as when a patient is nothing by mouth (NPO), and notify the provider of the potential need to adjust insulin accordingly. Nursing should also monitor trends in the blood glucose levels throughout the day and act as an advocate for patients receiving sliding-scale insulin with inadequate glycemic control by coordinating with providers and pharmacists and requesting a basal-bolus insulin regimen.

\section{Education}

Despite the evidence that basal-bolus insulin offers better glycemic control in the inpatient setting, sliding-scale insulin is still widely prescribed and administered more often to diabetic patients [7-9]. Therefore, healthcare providers may benefit from education that reviews the benefits of basal-bolus insulin as well as the disadvantages of sliding-scale insulin. It has been suggested that sliding-scale insulin is ordered among providers due to its convenience; however, the disadvantages of the practice should be presented to providers in order to improve patient safety and care.

Hypoglycemia is a potential complication associated with strict glycemic control and has the capacity to cause seizures, coma, or even death [5]. While only Umpierrez et al. [9], demonstrated that patients receiving basal-bolus insulin were more likely to experience a hypoglycemic episode, it may be valuable to provide an education review to nursing staff regarding the signs and symptoms of hypoglycemia so that it is readily recognized to avoid further adverse events. 


\section{Healthcare policy}

The Joint Commission (TJC) offers an Inpatient Diabetes Certification Program for hospitals who possess certain exceptional qualities such as written blood glucose monitoring protocols, education for patients on diabetes self-management, and program champions for staff education [14]. Hospitals who are certified are recognized to promote better patient outcomes and have the capabilities to meet the needs of the patients by following the clinical practice recommendation put forth by the American Diabetes Association [14]. Recently, the American Diabetes Association [15] published an article that strongly discouraged the sole use of sliding-scale insulin for diabetic inpatients due to poor glycemic control and increased rates of complications associated with its use. A recommendation to TJC would be to not offer certification to hospitals who are not compliant with ordering basal-bolus insulin and who manage their patients with sliding-scale insulin, as this practice is associated with ineffective blood glucose control and poor patient outcomes.

\section{Future research}

Further research that includes patients receiving certain medications such as corticosteroids as well as those with diagnoses that were excluded in the articles reviewed may be valuable. Not examining the relationship between insulin regimens in populations such as those with renal failure, heart failure, hepatic disease, or undergoing cardiac surgery suggests that these results are not applicable to a considerable amount of patients.

Given the data that supports basal-bolus insulin is more effective than sliding-scale insulin in managing blood glucose levels, it would be interesting to conduct further research on the barriers or reasoning behind why providers do not order the basal-bolus insulin regimen more frequently. Umpierrez et al. [8] reported that sliding-scale insulin is the insulin regimen of choice in acute care with less than one-half of patients being prescribed basal-bolus insulin. Conducting research to determine why providers choose the suboptimal treatment plan for managing blood glucose may allow researchers to address potential misconceptions regarding the 2 types of insulin regimens and promote the use of basal-bolus insulin.

\section{References}

[1] Centers for Disease Control and Prevention. Available at: https://www.cdc.gov/chronicdisease/ resources/publications/aag/diabetes.htm, accessed March 2017.

[2] AR Gosmanov. A practical and evidence-based approach to management of inpatient diabetes in non-critically ill patients and special clinical populations. J. Clin. Transl. Endocrinol. 2016; 5, 1-6.

[3] V Magaji and JM Johnston. Inpatient management of hyperglycemia and diabetes. Clin. Diabetes $2011 ; 29,3-9$.

[4] JG Timmons, SG Cunningham, CA Sainsbury and GC Jones. Inpatient glycemic variability and long-term mortality in hospitalized patients with type 2 diabetes. J. Diabetes Complications 2017; 31, 479-82.

[5] JA Johnston and ERV Horn. The effects of correction insulin and basal insulin on inpatient glycemic control. Medsurg. Nurs. 2011; 20, 187-93.

[6] BM Melnyk and E Fineout-Overholt. Evidence-based Practice in Nursing \& Healthcare: A Guide to Best Practice. $3^{\text {rd }}$ eds. Lippincott Williams \& Wilkins, Philadelphia, 2015, p. 91-2.

[7] HL Rymaszewski and S Breakwell. A retrospective review of sliding scale vs. basal/bolus insulin protocols. J. Nurs. Pract. 2013; 9, 214-8.

[8] GE Umpierrez, D Smiley, A Zisman, LM Prieto, A Palacio, M Ceron and R Mejia. Randomized study of basal-bolus insulin therapy in the inpatient management of patients with type 2 diabetes (RABBIT 2 trial). Diabetes Care 2007; 30, 2181-6.

[9] G Umpierrez, D Smiley, S Jacobs, L Peng, A Temponi, P Mulligan and M Rizzo. Randomized study of basal-bolus insulin therapy in the inpatient management of patients with type 2 diabetes undergoing general surgery (RABBIT 2 surgery). Diabetes Care 2011; 34, 256-61. 
http://wjst.wu.ac.th

[10] HH Zaman, V Permalu and SR Vethakkan. Sliding-scale versus basal-bolus insulin in the management of severe or acute hyperglycemia in type 2 diabetes patients: A retrospective study. Plos One 2014; 2014, e106505.

[11] S Clement, SS Braithwaite, MF Magee, A Ahmann, EP Smith, RG Schafer and IB Hirsh. Management of diabetes and hyperglycemia in hospitals. Diabetes Care. 2004; 27, 553-591.

[12] MB Christensen, A Gotfredsen and K Norgaard. Efficacy of basal-bolus insulin regimens in the inpatient management of non-critically ill patients with type 2 diabetes: A systematic review and meta-analysis. Diabetes Metab. Res. Rev. 2017; 33, e2885.

[13] YY Lee, YM Lin, WJ Leu, MY Wu, JH Tseng, MT Hsu, CS Tsai, AT Hsieh and K Tam. Slidingscale insulin used for blood glucose control: A meta-analysis of randomized controlled trials. Metab. Clin. Exp. 2015; 64, 1183-92.

[14] The Joint Commission. Available at: https://www.jointcommission.org/certification/ inpatient_diabetes.aspx, accessed March 2017.

[15] American Diabetes Association. Diabetes care in the hospital. Diabetes Care 2016; 39, 99-104. 